\title{
Nonspecific Interstitial Pneumonia/Fibrosis Completely Recovered by Adding Cyclophosphamide to Corticosteroids
}

\author{
Nobuki Nanki, Jiro FujtTA*, Yasufumi Yamaj, Hiroya Maeda, Taichi Kurose**, Mitsumasa KaJI**, \\ Katashi SatoH***, Katsuya Miyatani****, Ichiro Yamadori*****, Yuji Ohtsuki****** and Toshihiko Ishida*
}

\begin{abstract}
Nonspecific interstitial pneumonia/fibrosis (NSIP) was first described by Katzenstein and Fiorelli in 1994 (Am J Surg Pathol 18: 136-147). Many reports have described that corticosteroids are effective for NSIP. We describe a case of group II idiopathic NSIP in whom cyclophosphamide was administered since the initial response to corticosteroids had been insufficient. Lung biopsy was performed by video-assisted thoracoscopic surgery and NSIP was diagnosed pathologically, clinically and radiologically. Although the initial response to corticosteroids was insufficient, interstitial infiltrates on chest computed tomography improved dramatically after adding intravenous cyclophosphamide followed by oral cyclophosphamide. This case demonstrates that the addition of cyclophosphamide to corticosteroids might be a useful treatment for patients with NSIP.

(Internal Medicine 41: 867-870, 2002)
\end{abstract}

Key words: treatment, chest computed tomography, lung biopsy, completely remission

\section{Introduction}

Nonspecific interstitial pneumonia/fibrosis (NSIP) was first described by Katzenstein and Fiorelli in 1994 (1). NSIP has also been reported to be associated with collagen vascular disorders (1).

Many reports have described that corticosteroids are effective for NSIP (1-9). In addition, although other immunosuppressants (such as cyclophosphamide or azathioprine), have been used in patients with NSIP in whom corticosteroids were ineffective, the role of these immunosuppressants in patients with NSIP has not been clarified $(1,5-9)$.
In the present report, we describe a case of NSIP group II in whom interstitial infiltrates on chest computed tomography (CT) were resolved by cyclophosphamide added to corticosteroid treatment.

\section{Case Report}

A 48-year-old woman; a licensed cook, attended the outpatient clinic of the Mitoyo General Hospital with a non-productive cough and fever. Although she was treated with antibiotics, her symptoms worsened and she went to the emergency department due to dyspnea; she was then admitted to our hospital. She had not experienced any risk factors for occupational or environmental exposure to toxic materials, nor did she have hypersensitivity pneumonitis. She was a non-smoker and her previous medical history was not remarkable. There were no abnormal findings on chest computed tomography which had been taken 3-years earlier. On physical examination, she was febrile and cyanotic with a respiratory rate of 25 per minute. Her blood pressure was $114 / 62 \mathrm{mmHg}$. She had tachycardia without arrhythmia. Bibasilar inspiratory fine crackles were ausculated. Laboratory data showed a normal blood cell count. The electrolyte, renal and liver test was normal except for AST (51 IU/l). LDH and CRP were elevated. Autoantibodies; antinuclear antibody, rheumatoid factor, anti-single stranded DNA antibody, anti-double stranded DNA antibody, anti-RNP antibody, anti-Sm antibody, anti-Scl70 antibody or anti-Jo1 antibody were all negative. Arterial blood gas analysis showed $\mathrm{PaCO}_{2}$ of $29.0, \mathrm{PaO}_{2}$ of 53.0 Torr, $\mathrm{HCO}_{3}{ }^{-}$of $23.6 \mathrm{mEq} / l$, and $\mathrm{SaO}_{2}$ of $90.8 \%$. Lung function studies could not be performed because of severe dyspnea. Chest X-ray and chest $\mathrm{CT}$ revealed areas of patchy parenchymal opacification as well as thickening of perivascular and peribronchial bundles those present bilaterally mainly in the middle and lower lung zones (Fig. 1A). Flexible bronchoscopy was performed on the day of ad-

From the Department of Respiratory Medicine, Mitoyo General Hospital, Kagawa, *the First Department of Internal Medicine, Kagawa Medical University, Kagawa, **the Department of Radiology, Mitoyo General Hospital, Kagawa, ***the Department of Radiology, Kagawa Medical University, Kagawa, ****the Department of Pathology, Mitoyo General Hospital, Kagawa, *****the Department of Pathology, National Okayama Medical Center, Okayama and $* * * * * *$ the Department of Pathology, Kochi Medical University, Kochi

Received for publication January 25, 2002; Accepted for publication May 14, 2002

Reprint requests should be addressed to Dr. Nobuki Nanki, the Department of Respiratory Medicine, Mitoyo General Hospital, 708 Himehama, Mitoyo, Kagawa 769-1695 

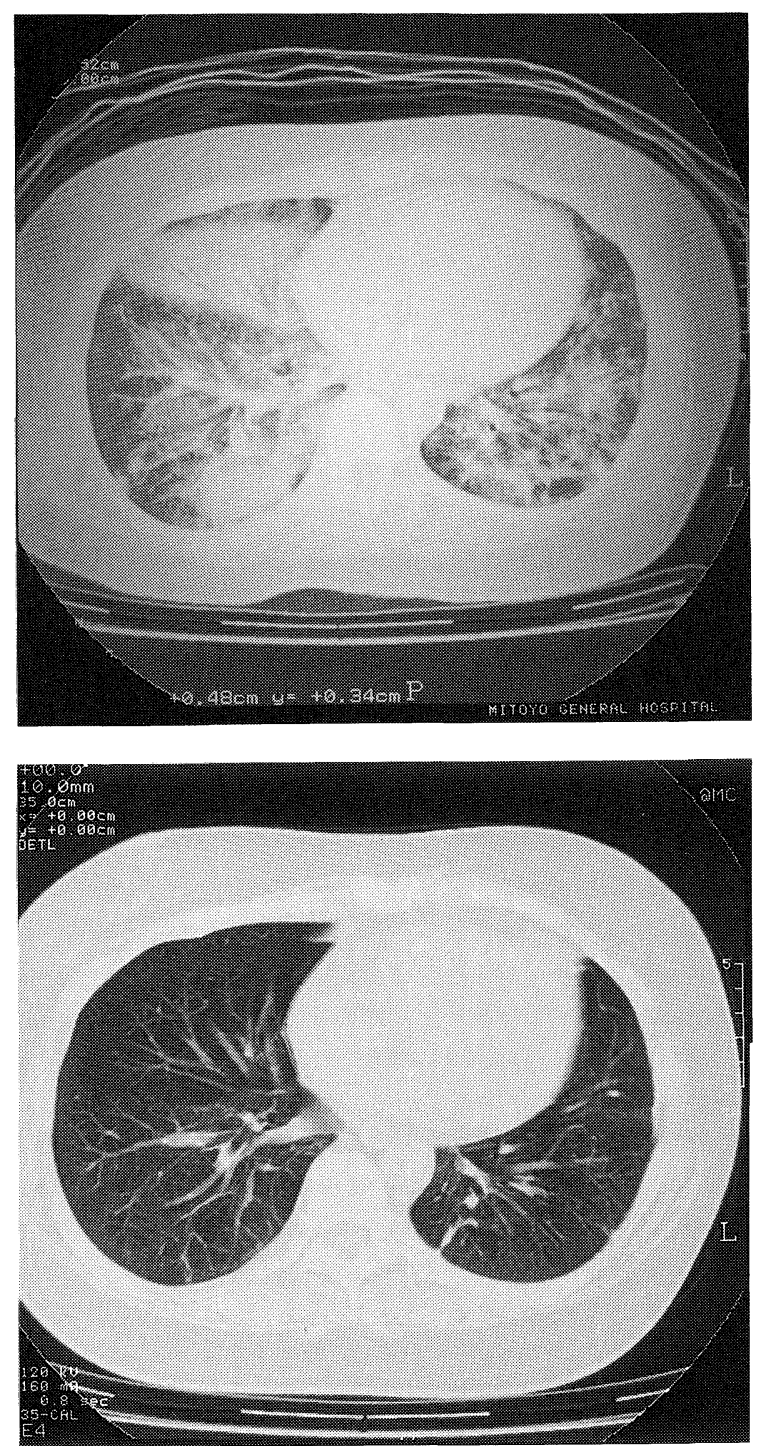

mission, and bronchoalveolar lavage (BAL) and transbronchial lung biopsy (TBLB) were performed from the right middle lobe. BAL fluid showed an increased cell number $(1,400$ cells/ $\mu \mathrm{l})$, lymphocytes predominance (59\%), eosinophil (7\%), neutrophil (17\%) and decreased CD4/CD8 ratio (0.31). Pathological evaluation of the specimen obtained by TBLB revealed compatibility with interstitial pneumonia. Based on radiological and pathological findings, acute interstitial pneumonia (AIP), bronchiolitis obliterance organizing pneumonia (BOOP), eosinophilic pneumonia, NSIP, or atypical pneumonia were considered. Therefore, intravenous methylprednisolone ( $1 \mathrm{~g} /$ day, for 3 days) followed by oral prednisolone and intravenous minocycline were administered (Fig. 2). Dyspnea and fever were improved and oral prednisolone was reduced gradually. However, at the time of prednisolone of $25 \mathrm{mg} / \mathrm{day}$, she became febrile and her chest computed tomography findings were not so improved (Fig. 1B). In addition, arterial blood gas analysis was deteriorated $\left(\mathrm{PaCO}_{2} 30.7\right.$ Torr, $\mathrm{PaO}_{2}$ 55.0 Torr). To make
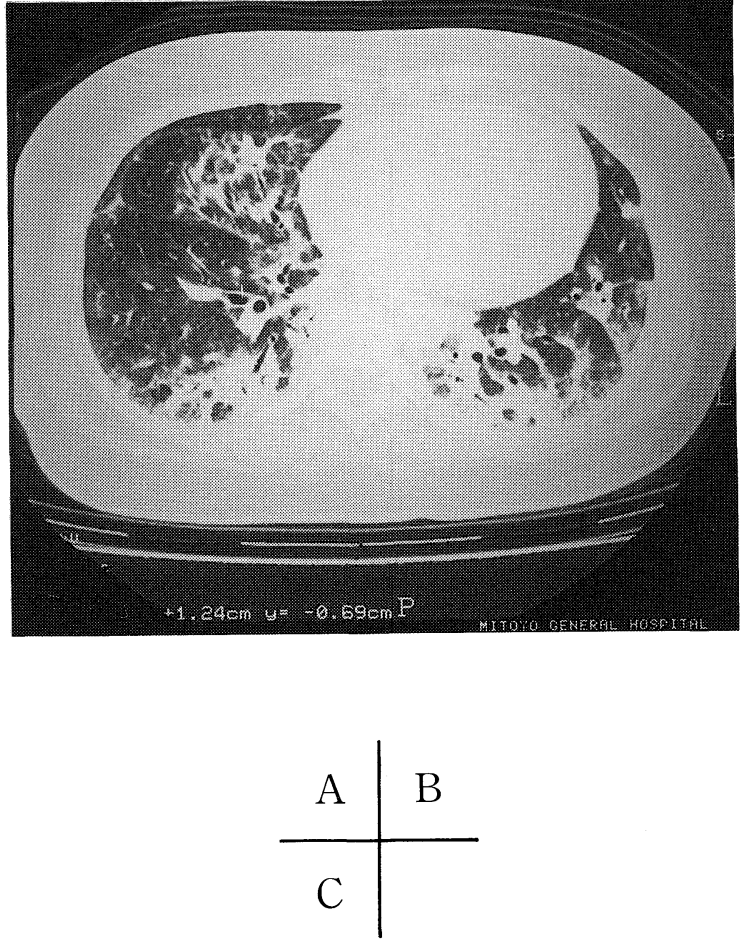

Figure 1. Chest CT findings of the present case. A) Mar. 4, 2001, B) April 2, 2001, C) Sept. 6, 2001.

the definite pathological diagnosis, lung biopsy was performed under video-assisted thoracoscopic surgery (VATS). Histological examination revealed intraluminal growth of connective tissues which were widely adherent to alveolar walls, accumulation of xanthoma cells in alveolar cavity, and diffuse infiltration of lymphocytes (Fig. 3). Since pathological, clinical and radiological diagnosis was NSIP group II, intravenous corticosteroids were administered again. However, while receiving prednisolone ( $25 \mathrm{mg} /$ day $)$, she became febrile again. In addition, arterial blood gas analysis was deteriorated again $\left(\mathrm{PaCO}_{2}\right.$ 33.4 Torr, $\mathrm{PaO}_{2}$ 58.2 Torr). Therefore, we added intravenous cyclophosphamide $(800 \mathrm{mg} /$ body $\times 3$, weekly) followed by oral cyclophosphamide ( $100 \mathrm{mg} /$ body). Three months after adding cyclophosphamide, her chest computed tomography revealed that interstitial infiltrates had dramatically improved (Fig. 1C). Her arterial blood gas analysis became normal which showed $\mathrm{PaCO}_{2}$ of $39.7, \mathrm{PaO}_{2}$ of 97.0 Torr, $\mathrm{HCO}_{3}{ }^{-}$of $26.4 \mathrm{mEq} / l$, and $\mathrm{SaO}_{2}$ of $97.7 \%$, and her pulmonary function test became nor- 

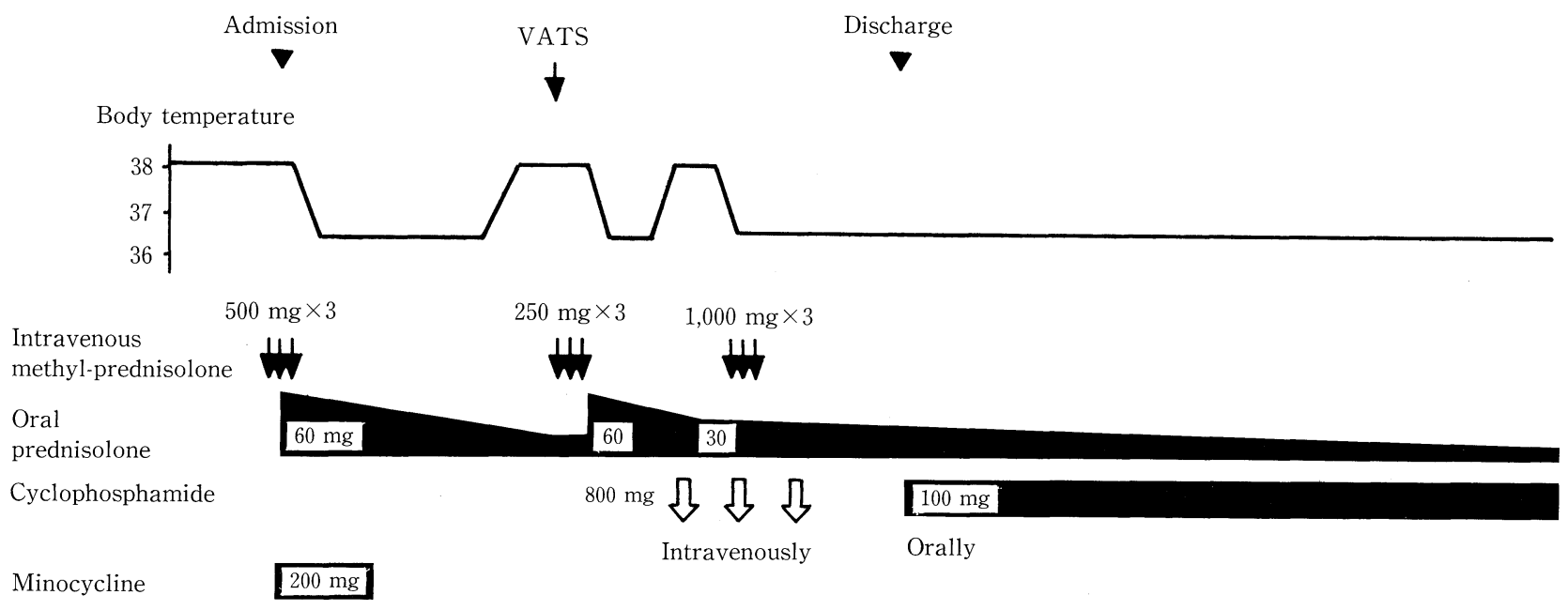

\begin{tabular}{|c|c|c|c|c|c|c|c|c|}
\hline Clarithromycin & & & & & & & & \\
\hline KL-6 (U/ml) & 1,610 & 2,070 & 1,740 & 970 & 1,320 & 1,250 & 624 & 402 \\
\hline SP-D (U/ml) & 303 & 191 & 222 & 84.8 & 112 & 82.3 & 27.3 & 26.2 \\
\hline $\mathrm{LDH}(\mathrm{IU} / l)$ & 553 & 237 & 243 & 221 & 227 & 368 & 325 & 301 \\
\hline CRP (mg/dl) & 4.92 & 0.19 & 0.37 & 0.16 & $<0.1$ & 0.12 & $<0.1$ & 0.11 \\
\hline
\end{tabular}

Figure 2. Clinical course of the present case. Normal values: KL-6: <500 U/ml, SP-D: <110 U/ml, LDH: 70-210 IU/ml, CRP: $<0.25 \mathrm{mg} / \mathrm{dl}$.

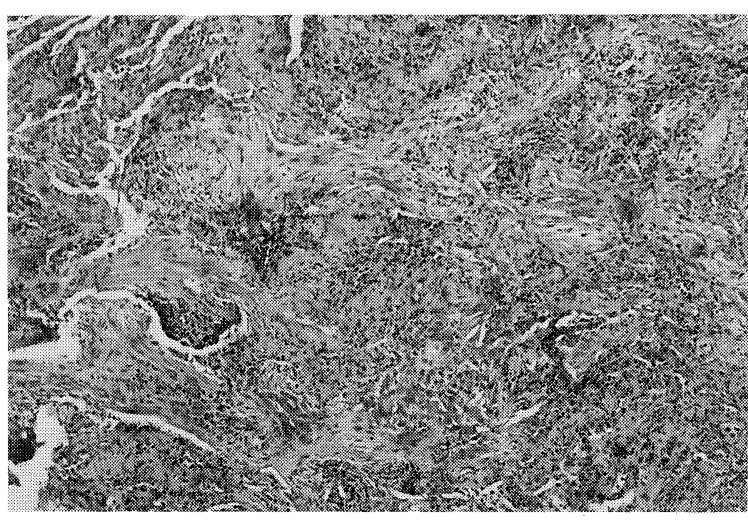

A

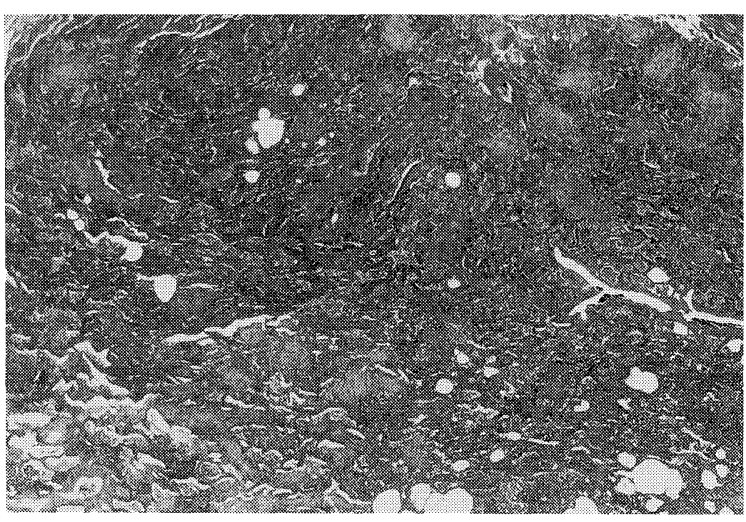

$\mathrm{B}$

Figure 3A. Histological findings of the present case. Pathological diagnosis was group II nonspecific interstitial pneumonia (HE stain, $\times 25)$. B) Histological findings of the present case. Pathological diagnosis was group II nonspecific interstitial pneumonia (HE stain, $\times 6.6$ ).

mal which showed per cent vital capacity of $87.7 \%$, and per cent forced expiratory volume in 1 second of $83.04 \%$.

\section{Discussion}

In this report, we described a case of idiopathic group II NSIP in whom interstitial infiltrates were resolved by adding 
NANKI et al

Table 1. Review of Clinical Features of Nonspecific Interstitial Pneumonia

\begin{tabular}{|c|c|c|c|c|c|c|c|c|c|c|}
\hline Katzenstein & 1994 & 64 & $26 / 38$ & 46 & $\mathrm{D} 80, \mathrm{C} 33, \mathrm{~F} 22$ & 8.1 & $\mathrm{G} 1: 31, \mathrm{G} 2: 24, \mathrm{G} 3: 9$ & 33 & $\mathrm{Cp} 6, \mathrm{~A} 2$ & $6 \mathrm{Di}$ \\
\hline Park & 1995 & 7 & $1 / 6$ & 55 & D100, C43 & 4 & $\mathrm{G} 1: 1, \mathrm{G} 2: 4, \mathrm{G} 3: 2$ & 7 & 0 & None \\
\hline Park & 1996 & & & & & & & & & \\
\hline Nagai & 1998 & 31 & $15 / 16$ & 57 & $\mathrm{R} 100, \mathrm{~F} 33$ & 2 & G1:2, G2:14, G3:15 & 31 & $8(\mathrm{Cp}$ or $\mathrm{A})$ & $2 \mathrm{Di}, 1 \mathrm{PD}, 5 \mathrm{PR}$ \\
\hline Cottin & 1998 & 12 & $6 / 6$ & 53 & D100, C67, G58, B42 & 44 & $\mathrm{G} 1: 3, \mathrm{G} 2: 8, \mathrm{G} 3: 1$ & 6 & $\mathrm{~A} 4, \mathrm{~A}+\mathrm{Cp} 1, \mathrm{~A}+\mathrm{M} 1, \mathrm{Cp} 1$ & $2 \mathrm{PD}, 5 \mathrm{PR}$ \\
\hline Fujita & 1999 & 24 & $7 / 17$ & 60 & C83, D71, F29 & 31 & $\mathrm{G} 2: 24$ & 7 & $\mathrm{Cp} 3$ & $1 \mathrm{PD}, 2 \mathrm{PR}$ \\
\hline Daniil & 1999 & 15 & $7 / 8$ & 43 & D100, C60 & 8 & G: not classifiable & 15 & $\mathrm{~A} 8, \mathrm{Cp} 2, \mathrm{Cs} 1$ & 2Di, 3PD, 6PR \\
\hline Present report & 2002 & 1 & $0 / 1$ & 48 & $\mathrm{~F}, \mathrm{C}, \mathrm{D}$ & 0.5 & G2 & 1 & 1 & $\mathrm{CR}$ \\
\hline
\end{tabular}

D: Dyspnea, C: Cough, F: Fever, S: Sputum, R: Respiratory symptom, G: General fatigue, B: Body weight loss. **G1: Groupe I, G2: Groupe II, G3: Groupe III. *** Number of cases in whom immunosuppressive agents as well as corticosteroids were used. Cp: Cyclophosphamide, A: Azathioprine, M: Methotrexate, Cs: cyclosporin. **** Di: Died, PD: Progressive disease, PR: Partial response, CR: Complete remission.

cyclophosphamide to corticosteroids. Although several immunosuppressants were used in patients with NSIP associated with collagen vascular disorders, this is the first report which describes the clinical course of an idiopathic NSIP patient in whom cyclophosphamide adding to corticosteroids was very effective.

NSIP was first described by Katzenstein and Fiorelli in 1994 (1). In the initial description of this pattern of interstitial pneumonia, NSIP was proposed as a diagnosis in specimens with findings that did not conform to the typical findings seen in usual interstitial pneumonia (UIP), desquamative interstitial pneumonia (DIP), AIP, or BOOP (1). In addition, it has been reported that NSIP is associated with collagen vascular disorders (1).

We reviewed the clinical features of NSIP described in several reports (Table 1). There have been many reports of patients with NSIP in whom corticosteroids or other immunosuppressants were used (1-10). Although several immunosuppressants (such as, cyclophosphamide or azathioprine), were used for patients with NSIP in whom initial response to corticosteroids was not sufficient, the usefulness of these immunosuppressants for NSIP has not been established (1, 5-9). Ogasawara et al have reported successful treatment with lowdose cyclosporin $\mathrm{A}(1 \mathrm{mg} / \mathrm{kg} / \mathrm{day})$ in a patient with interstitial pneumonitis associated with Sjögren's syndrome who was resistant to corticosteroids (11). In addition, in NSIP associated with collagen vascular diseases, cyclophosphamide is frequently used. However, there has been no report that describes a case of idiopathic NSIP in whom interstitial infiltrates were resolved by adding cyclophosphamide to corticosteroids. The present case, however, demonstrates that cyclophosphamide added to corticosteroid therapy might be a useful treatment for patients NSIP. Further studies are necessary to evaluate the role of cyclophosphamide in the treatment of NSIP.

\section{References}

1) Katzenstein AL, Fiorelli RF. Nonspecific interstitial pneumonia/fibrosis. Histologic features and clinical significance. Am J Surg Pathol 18: 136147, 1994.

2) Park JS, Lee KS, Kim JS, et al. Nonspecific interstitial pneumonia with fibrosis: radiographic and CT findings in seven patients. Radiology 195: 645-648, 1995.

3) Park CS, Jeon JW, Park SW, et al. Nonspecific interstitial pneumonia/ fibrosis: clinical manifestations, histologic and radiologic features. Korean J Intern Med 11: 122-132, 1996.

4) Kim TS, Lee KS, Chung MP, et al. Nonspecific interstitial pneumonia with fibrosis: high-resolution CT and pathologic findings. AJR Am J Roentgenol 171: 1645-1650, 1998.

5) Bjoraker JA, Ryu JH, Edwin MK, et al. Prognostic significance of histopathologic subsets in idiopathic pulmonary fibrosis. Am J Respir Crit Care Med 157: 199-203, 1998.

6) Nagai S, Kitaichi M, Itoh H, Nishimura K, Izumi T, Colby TV. Idiopathic nonspecific interstitial pneumonia/fibrosis: comparison with idiopathic pulmonary fibrosis and BOOP. Eur Respir J 12: 1010-1019, 1998.

7) Cottin V, Donsbeck AV, Revel D, Loire R, Cordier JF. Nonspecific interstitial pneumonia. Individualization of a clinicopathologic entity in a series of 12 patients. Am J Respir Crit Care Med 158: 1286-1293, 1998.

8) Fujita J, Yamadori I, Suemitsu I, et al. Clinical features of non-specific interstitial pneumonia. Respir Med 93: 113-118, 1999.

9) Daniil ZD, Gilchrist FC, Nicholson AG, et al. A histologic pattern of nonspecific interstitial pneumonia is associated with a better prognosis than usual interstitial pneumonia in patients with cryptogenic fibrosing alveolitis. Am J Respir Crit Care Med 160: 899-905, 1999.

10) Fujita J, Yamadori I, Bandoh S, et al. Clinical features of three fatal cases of non-specific interstitial pneumonia. Intern Med 39: 407-411, 2000.

11) Ogasawara H, Sekiya M, Murashima A, et al. Very low-dose cyclosporin treatment of steroid-resistant interstitial pneumonitis associated with Sjögren's syndrome. Clin Rheumatol 17: 160-162, 1998. 